EPSC Abstracts

Vol. 14, EPSC2020-746, 2020, updated on 07 Jan 2021

https://doi.org/10.5194/epsc2020-746

Europlanet Science Congress 2020

(C) Author(s) 2021. This work is distributed under

the Creative Commons Attribution 4.0 License.

\title{
Atypical landslides in the Nilosyrtis region of Mars
}

Anthony Guimpier, Susan Conway, and Nicolas Mangold

Laboratoire de Planétologie et Géodynamique CNRS UMR6112, University of Nantes, France (anthony.guimpier@univ-nantes.fr)

Introduction: This study focuses on four unusual landslides located inside a $25 \mathrm{~km}$ diameter impact crater located in Nilosyrtis Mensae (Fig.1). Our initial study revealed that one of these landslides bears close similarity to a mudslide on Earth [1]. We performed a detailed morphological analysis and a comparison with terrestrial analogues to better understand their formation mechanism. We also consider the possible role of volatiles in their formation.

Approach: We used the Colour and Stereo Surface Imaging System (CaSSIS) [2] and ConTeXt imager (CTX) with a resolution of $6 \mathrm{~m} /$ pix and $5 \mathrm{~m} / \mathrm{pix}$, respectively, to carry out a regional study, while the High Resolution Imaging Science Experiment (HiRISE) images with a resolution of 25-50 $\mathrm{cm} /$ pix were used to carry out a detailed study of the landslides. We also used the HiRISE data to make Digital Elevation Models (DEMs) at $2 \mathrm{~m} /$ pix produced using the standard workflow in ISIS and SocetSet [3]. 


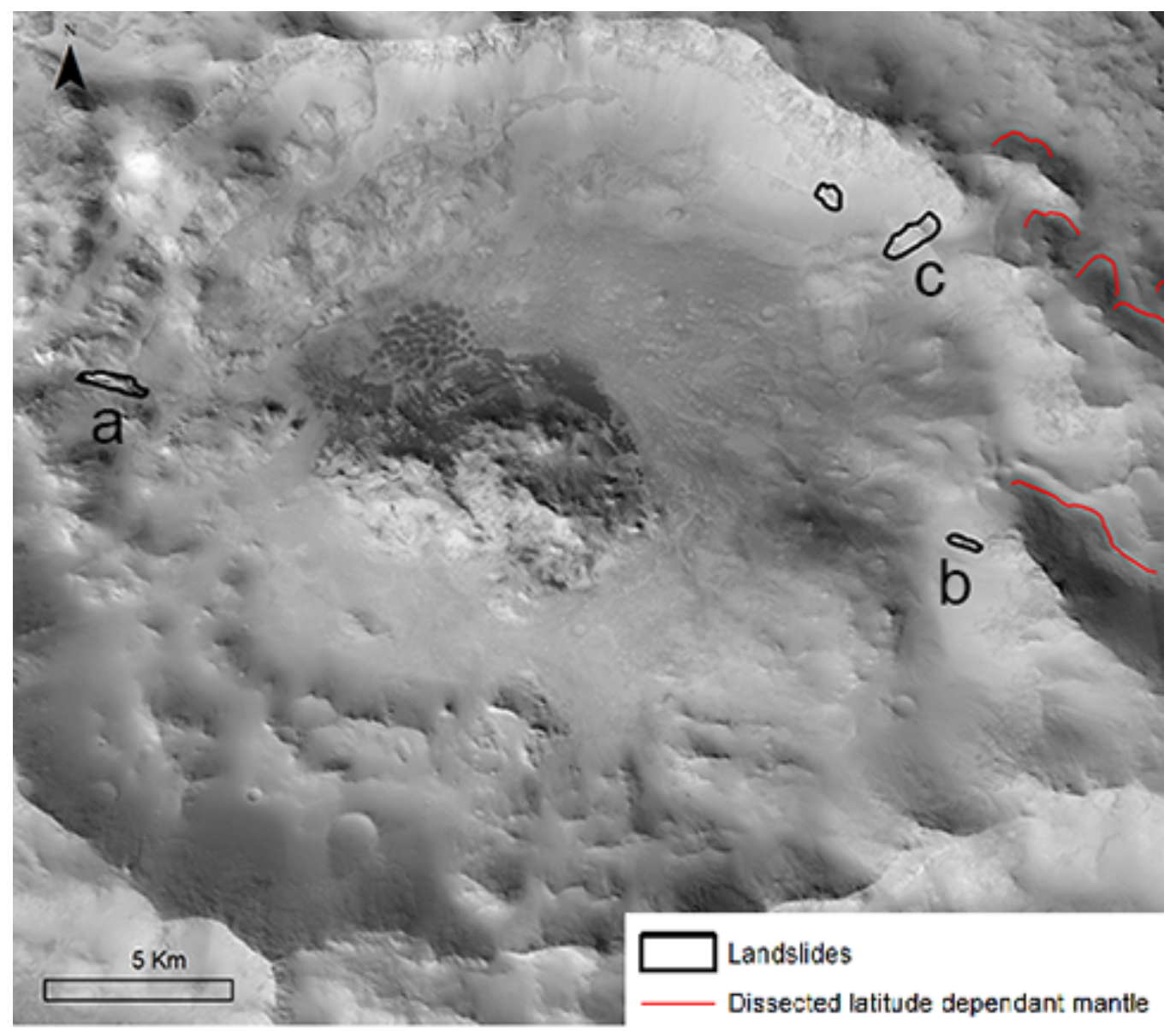

Figure 1 - General view of the study area with the 4 landslides in the Nilosyrtis Mensae area, at about $27^{\circ} \mathrm{N}$ latitude, $76^{\circ} \mathrm{W}$ longitude. a, b and c show the location of landslides seen in figure 2. CTX images K11_057796_2076 and B11_01409_2058 (NASA/JPL/MSS)

Morphological analysis results: The host crater has a rounded rim and highly degraded ejecta suggesting a Hesperian to Early Amazonian age. The crater shows some evidence of dissected latitude dependant mantle on pole-facing slopes on the outer crater walls (Fig.1) -

this is an ice-rich deposit which is thought to date to a few tens of Ma [4]. There are also 200 wellpreserved rockfall tracks on the eastern crater wall, indicating that the interior of the crater has had episodes of recent destabilization during the late Amazonian. The landslides have the following features in common: (1) a sliding surface with an angle between 20 and $25^{\circ} ;(2)$ an irregular, welldefined erosion zone; (3) compression ridges on the deposit; (4) a lobate deposit margin; and (5) lateral levees on 3 out of the 4 landslides with a thickness between 5 and 25 meters. In addition, on two of the four landslides, there are secondary failure scars within the main landslide deposit, suggesting reactivation, as shown in in Figure 3a. In addition, the overall morphology of these four landslides suggests a late Amazonian formation-age, based on their pristine appearance and the absence of impact craters on the deposit. The broadly similar ages of the landslides and the degraded latitude dependent mantle suggests a potential link. 


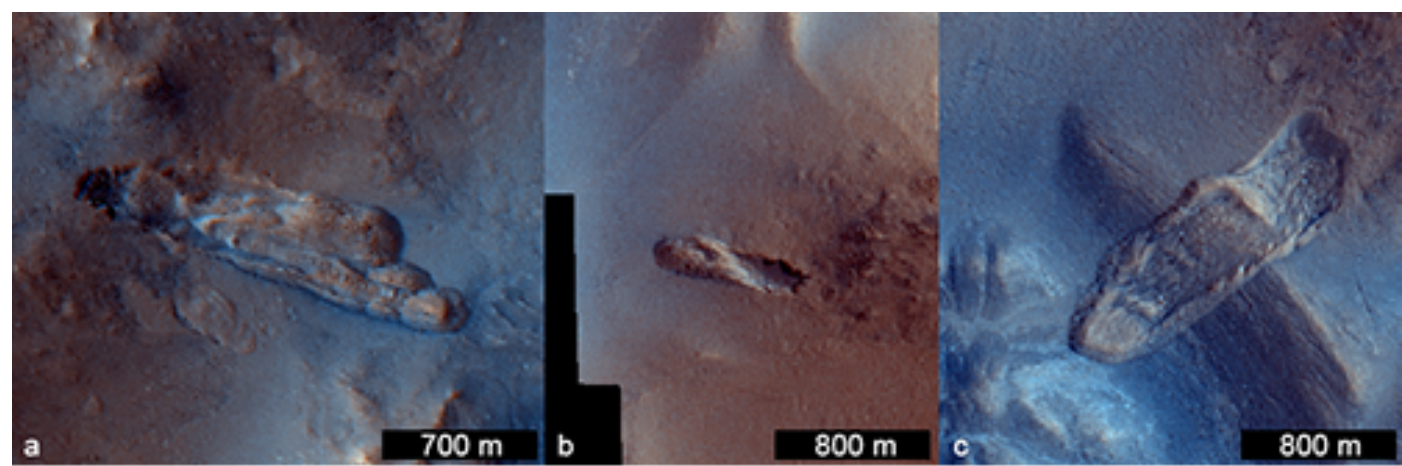

Figure 2 - CaSSIS images of three of the four landslides located in the Nilosyrtis Mensae region. MY35_008346_155 \& MY35_008751_028, RGB composition using PAN and BLU filters (ESA/Roscosmos/ŪniBe).

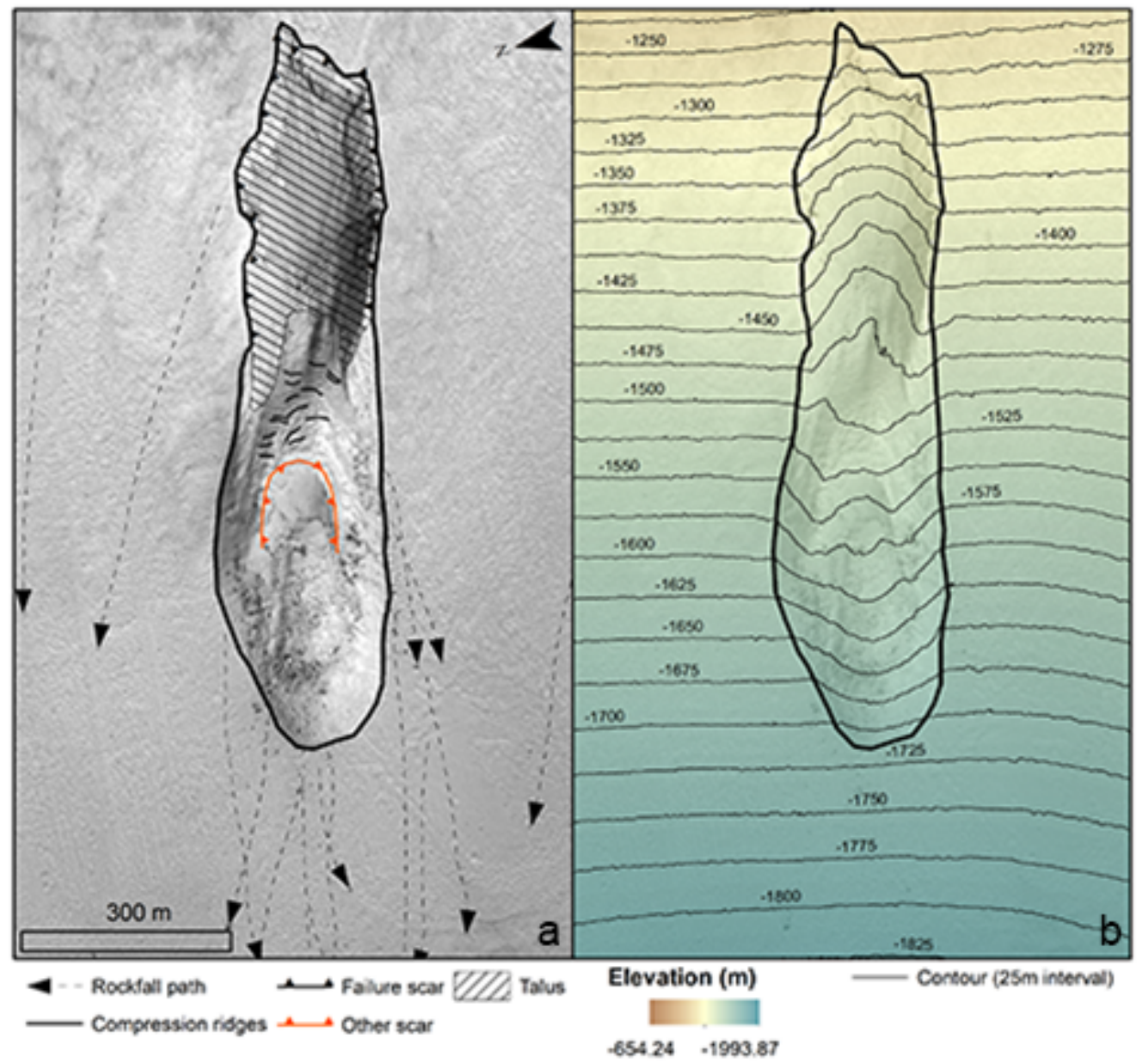

Figure 3 - (a) Map of the main structures and (b) elevation map of one of the studied landslides. HiRISE: ESP_057110_2075.

(NASA/JPL/UofA)

Terrestrial comparison: We conducted a morphological and rheological comparison with terrestrial analogues to better understand the flow dynamics of these martian landslides. We found that these landslides are most comparable with terrestrial earthflows, because they also: (1) occur on low surface slopes, (2) have lateral levees (3) have high relief lobate flow fronts and (4) possess similar 
material yield strength values around $100 \mathrm{kPa}$ [5]. We estimated the yield strength of our martian landslides to be $1-100 \mathrm{kPa}$ by using the cross-profile morphology of the flows as described in [6].

Conclusions: (1) We see numerous rockfall tracks showing the presence of recent instabilities in this region. (2) The observation of dissected latitude dependant mantle reveals ice has recently been present in the ground in this region. (3) The comparison that we made between the martian landslides and terrestrial analogues reveals similarity with earthflows in terms of morphology and rheology. (4) Liquid water is key to allowing earthflows to move on Earth, and the dissected latitude dependant mantle near the impact crater reveals the presence of ice in the past. We therefore propose that ice could have been involved in the formation of these landslides.

Acknowledgements: This thesis is funded by a ministerial grant. We are grateful for the support of the Programme National de Planétologie and the French Space Agency CNES. The authors thank the spacecraft and instrument engineering teams for the successful completion and operation of CaSSIS. CaSSIS is a project of the University of Bern funded through the Swiss Space Office via ESA's PRODEX program. The instrument hardware development was also supported by the Italian Space Agency (ASI) (ASI-INAF agreement no. I/018/12/0), INAF/ Astronomical Observatory of Padova, and the Space Research Center (CBK) in Warsaw. Support from SGF (Budapest), the University of Arizona (LPL) and NASA are also gratefully acknowledged.

References: [1] Guimpier et al. (2020) PSS, submitted. [2] N. Thomas et al. (2017) Space Sci. Rev., 212, 1897-1944. [3] R.L. Kirk et al. (2008) JGR, 113. [4] R.E. Milliken et al. (2003) JGR, 108, E6. [5] A.L. Nereson and N.J. Finnegan (2015) AGU Fall Meet. Abstr., EP41C-0943. [6] D.M. Pyle and J.R. Elliott (2006) Geosphere, 2, 253-268. 